\title{
RNF6 Targeted by miR-26a-5p Protects Pancreatic $\beta$-Cell Function Against Type 2 Diabetes
}

\author{
Fan Yang ${ }^{1} *$ \\ Shengxun Zhao ${ }^{2, *}$ \\ Xuyan Zhang ${ }^{3}$ \\ Sheng Ding ${ }^{3}$ \\ Yancheng $\mathrm{Xu}{ }^{\prime}$ \\ 'Department of Endocrinology, Wuhan \\ University Zhongnan Hospital, Wuhan, \\ 430000, Hubei, People's Republic of \\ China; ${ }^{2}$ Department of Geriatrics, The \\ First Hospital of Wuhan, Wuhan, 430000 , \\ Hubei, People's Republic of China; \\ ${ }^{3}$ Department of Endocrinology, The \\ Central Hospital of Wuhan, Wuhan, \\ 4300 I4, Hubei, People's Republic of \\ China
}

*These authors contributed equally to this work
Correspondence: Yancheng $\mathrm{Xu}$ Department of Endocrinology, Wuhan University Zhongnan Hospital, No. 169, Donghu Road, Wuchang District, Wuhan, 430000, Hubei, People's Republic of China Tel +86 I3907II6967

Email yanchengxu26@163.com
Background: Type 2 diabetes (T2D) is characterized by progressive $\beta$-cell dysfunction. Regulatory microRNAs (miRNAs) may be associated with this.

Methods: Serum miR-26a-5p and RNF6 levels were detected in T2D patients and healthy volunteers via qRT-PCR. Subsequently, the role of specific dysregulated miR-26a-5p or RNF6 in regulating insulin content, cell proliferation, and apoptosis was studied in INS-1 cells. The targeting correlation between miR-26a-5p and RNF6 was detected using a luciferase assay.

Results: RNF6 expression was significantly decreased in T2D individuals and INS-1 cells treated with high glucose, while miR-26a-5p expression was increased. In INS-1 cells, RNF6 overexpression or miR-26a-5p downregulation significantly increased insulin content and secretion, induced proliferation, and inhibited apoptosis. RNF6 has been identified as an miR-26a-5p target, which negatively regulates RNF6 to worsen INS-1 cell function.

Conclusion: RNF6 promoted insulin secretion and induced cell proliferation in INS-1 cells. This may be related to miR-26a-5p targeting and negatively regulating T2D pathogenesis.

Keywords: RNF6, miR-26a-5p, pancreatic, $\beta$-cell, type 2 diabetes, INS-1

\section{Introduction}

Type 2 diabetes (T2D) is the most common metabolic syndrome, characterized by impaired insulin secretion from pancreatic $\beta$-cells and peripheral insulin resistance. ${ }^{1}$ In 2019 , more than 4.5 billion adults worldwide were diagnosed with diabetes, 90 $95 \%$ of whom suffered from T2D, which has become a global health problem and places a huge burden on the social healthcare system. ${ }^{2,3}$ At present, clinical intervention for T2D mainly depends on insulin supplementation or antidiabetic drugs, but it cannot be eradicated. ${ }^{4}$ Studies have shown that genetic, epigenetic, and environmental factors interact and contribute to the development of diabetes. ${ }^{5}$ Therefore, deepening our understanding of T2D's pathological processes and exploring the genes at play may provide new ideas for T2D treatment.

Ring finger protein 6 (RNF6), located on chromosome 13q12.13, is an RNF family RING-type E3 ubiquitin-protein ligase related to target protein degradation and ubiquitination., ${ }^{6,7}$ Several studies have shown that RNF6 helps regulate cell proliferation, metabolism, and apoptosis. ${ }^{8,9}$ In addition, a study on the long-term memory of endothelial cells in response to hyperglycemic stress under normal blood glucose found that RNF6 was a hyperglycemia-induced metabolic memoryrelated gene. ${ }^{10}$ However, the effects and mechanisms of RNF6 in T2D have rarely been reported. 
Non-coding genes increase the risk of diabetes by modifying gene regulation, which alters protein production and function. ${ }^{11}$ MicroRNAs (miRNAs), which inhibit gene expression by targeting complementary mRNA regions and inhibiting protein translation, are non-coding RNAs. ${ }^{12}$ Accumulating evidence suggests that miRNAs are powerful cell activity regulators of cell growth, differentiation, development, and apoptosis and are associated with various diseases, including metabolic disorders. ${ }^{13}$ Plasma miR-26b-5p may be involved in T2D pathogenesis. Studies have shown that miR-26b-5p was only detected in T2D patients compared with healthy controls. ${ }^{14}$ In addition, miR-26b-5p was significantly downregulated in plasma samples from patients with T2D after intervention with hypoglycemic drugs. ${ }^{15}$ Furthermore, miR-26b can accelerate the progression of gestational diabetes by inhibiting the PI3K/Akt signaling pathway. ${ }^{16}$ Notably, one study revealed that miR-26b targets the RNF6 3'UTR and suppresses RNF6 expression in rifampin-treated $\mathrm{HCC}$ cells. ${ }^{17}$ However, there are no studies on how miR-26b -5 p/RNF6 affects T2D progression.

In our study, we explored the specific relationship between miR-26b-5p/RNF6 and T2D. We investigated the effects of miR-26b-5p/RNF6 on insulin content, insulin secretion, proliferation, and apoptosis in insulin-secreting $\beta$-cells. This study aimed to develop an effective T2D treatment.

\section{Methods}

\section{Blood Sample Collection}

This study was conducted per the Declaration of Helsinki and was approved by the Ethics Committee of Wuhan University Zhongnan Hospital (Approval number: NFEC2019-006). Written informed consent was obtained from all participants before enrollment. Blood samples were collected from $34 \mathrm{~T} 2 \mathrm{D}$ patients and 34 healthy volunteers in our hospital according to the World Health Organization's diagnostic criteria for T2D. ${ }^{18}$ Patients with severe liver and kidney disease, acute heart failure, or malignant tumors were excluded from the study. Venous blood samples were centrifuged at $3000 \mathrm{r} / \mathrm{min}$ for $10 \mathrm{~min}$. The upper supernatant was collected and sub-packaged in different Eppendorf tubes, and the sub-packaged plasma was frozen at $-80^{\circ} \mathrm{C}$ for the real-time quantitative PCR (qRT-PCR) assay.

\section{Cell Culture}

The pancreatic $\beta$-cell line INS- 1 from the Chinese Academy of Sciences Cell Bank (China) was placed in a DMEM medium containing $5.5 \mathrm{mM}$ glucose (normal). Additional INS-1 cells were added to the $25 \mathrm{mM}$ glucose (model) to construct a hyperglycemia-induced cell model. In addition, $10 \%$ FBS, penicillin (100 U/mL), and Streptococcus spp. (100 $\mathrm{U} / \mathrm{mL}$ ) were added to the medium and stored in a humidified incubator at $37^{\circ} \mathrm{C}$ with $5 \% \mathrm{CO}_{2}$. After $24 \mathrm{~h}$ of culture, the INS- 1 cells were used to study cell transfection, insulin secretion, insulin content, cell proliferation, and apoptosis.

\section{Cell Transfection}

siRNA (si-RNF6), pcDNA3.1 (pcDNA3.1-RNF6), and the corresponding negative RNF6 controls (si-NC and pcDNA3.1, respectively) were obtained from GeneChem (Shanghai, China). miR-26a-5p inhibitor, mimic, and corresponding negative controls (inhibitor-NC and mimic-NC, respectively) were obtained from Switchgear Genomics (USA). Using Lipofectamine 2000 (Invitrogen, USA), 50 nM siRNA, $75 \mathrm{ng}$ pcDNA3.1, $100 \mathrm{nM}$ inhibitor, and 100 $\mathrm{nM}$ mimic were transfected into INS-1 cells. Transfection efficiency was measured $48 \mathrm{~h}$ after transfection.

\section{qRT-PCR Assay}

miRNA was extracted from blood and cells using the PAXgene Blood miRNA Kit (Qiagen, USA) and the miRNeasy Mini Kit (Qiagen), respectively. The samples were purified and quantified using a spectrophotometer (Gilford Products Laboratories, USA), followed by reverse transcription using the miRcute miRNA First-strand cDNA Synthesis Kit (Tiangen, China). qRT-PCR was carried out in the iQ5TM Bio-Rad system (BioRad, USA) per manufacturer instructions on miRcute miRNA qPCR detection kit (Tiangen). The relative miRNA expression was calculated using the formula $2^{-\Delta \Delta \mathrm{Ct}},{ }^{19}$ and U6 was used as an internal control.

Total RNA was extracted using the Qiagen QIAamp RNA Blood Mini Kit (Qiagen). Total RNA was reverse transcribed into cDNA using the iScript cDNA Synthesis Kit (BioRad, USA), and qRT-PCR was performed using SYBR Green Master PCR Mix (Thermo Fisher Scientific, USA) with GAPDH as an internal reference. Table 1 lists the primer sequences used in this study.

\section{Western Blot Assay}

Cells were treated with RIPA buffer (Beyotime, China) to obtain the total cell proteins, which were quantified using the micro bicinchoninic acid (BCA) method. Proteins (50 $\mu \mathrm{g})$ were separated by SDS-PAGE, transferred to PVDF membranes, and sealed with $5 \%$ skimmed milk at $37^{\circ} \mathrm{C}$ for $2 \mathrm{~h}$. Then, the membrane was combined with RNF6 
Table I The Primers Used in the Presence Work

\begin{tabular}{|l|c|r|}
\hline Targets & Primers & Sequences (5' to 3') \\
\hline Human miR- & Forward & 5'-UCCAUAAAGUAGGAAACACUACA-3' \\
Human U6 & Reverse & 5'-CAGUACUUUUGUGUAGUACAA-3' \\
Human RNF6 & Reverse & 5'-CTCGCTTCGGCAGCACATATACT-3' \\
& 5'-ACGCTTCACGAATTTGCGTGTC-3' & 5'-AGAAGATGGCAGCAAGAGCG-3' \\
Human & Reverse & 5'-TCAAGTCAGGCTGAGATGCTAGT-3' \\
GNAS & Rerward & 5'-CCATGGGCTGCCTCGGAAACA-3' \\
Human & Forward & 5'-TTAAGCAAGCGGAAGGGAAGAAA-3' \\
GAPDH & Reverse & 5'-TGGTGAAGACGCCAGTGGA-3' \\
Rat miR-26a- & Forward & 5'-UUCAAGUAAUCCAGGAUAGGCU-3' \\
5p & Reverse & 5'-GTGCAGGTCCGAGGT-3' \\
Rat U6 & Forward & 5'-CTCGCTTCGGCAGCACA-3' \\
Rat RNF6 & Reverse & 5'-AACGCTTCACGAATTTGCGT-3' \\
Rat GAPDH & Forward & 5'-CAGTGGCGTGCCTTAGTTCT-3' \\
& Reverse & 5'-AAGGCCTCTACGCCCTCTAA-3' \\
5'-GGAGCGAGATCCCTCCAAAAT-3' & 5'-GGCTGTTGTCATACTTCTCATGG-3' \\
\hline
\end{tabular}

Abbreviations: RNF6, Ring finger protein 6; GNAS, GNAS complex locus.

(ab204506; Abcam, USA) or GAPDH (ab9484; Abcam) antibodies overnight at $4{ }^{\circ} \mathrm{C}$. The secondary antibody was horseradish peroxidase-labeled goat anti-rabbit $\operatorname{IgG}$ (ab205718; Abcam). The ECL method was used to detect the signal, the UVI gel imaging system was used to collect the image, and Image $\mathrm{J}$ software (Media Cybernetics, USA) was used to analyze the gray value.

\section{ELISA Assay}

Insulin secretion levels were determined using an insulin ELISA Kit (RayBiotech, USA). INS-1 cells were collected after $48 \mathrm{~h}$ of transfection and incubated in a glucose-free medium for $1 \mathrm{~h}$. Cells were then treated with $3.3 \mathrm{mmol} / \mathrm{L}$ or $16.7 \mathrm{mmol} / \mathrm{L}$ glucose for $2 \mathrm{~h}$. The insulin secretion in the supernatant was determined. ${ }^{20}$

Insulin content was determined using radioimmunoassay. Cells were collected and suspended in $300 \mathrm{~mL}$ of distilled water, then homogenized on ice using an ultrasound. The homogenate was treated with acidic ethanol, and the treatment was frozen/thawed and centrifuged. The insulin level in the supernatant was determined using an ELISA Kit (Meridia, Sweden), and the DNA concentration was quantified using DNA PicoGreen assay. ${ }^{21}$

\section{EdU Assay}

INS-1 cell proliferation was measured using an EdU Kit (RiboBio, China). Briefly, the cells were collected and co- incubated with EdU, and a 4\% PFA fixation solution was added to fix the cells. The cells were then stained for 30 min in a $1 \times$ Apollo reaction cocktail, and then incubated for 30 min with DAPI. Finally, the cells were observed and photographed using a fluorescence microscope (Leica, Germany). Nuclei labeled with EdU and DAPI were positive.

\section{Apoptosis Assay}

The transfected cells were resuspended in $500 \mu \mathrm{L}$ of $1 \times$ binding buffer. Apoptosis levels were measured using an annexin V FITC/PI Assay Kit (BD, USA). The cell suspension was supplemented with $5 \mu \mathrm{L}$ V-FITC and $5 \mu \mathrm{L}$ PI. After incubation at $25^{\circ} \mathrm{C}$ in darkness for $5 \mathrm{~min}$, flow cytometry analysis was performed using BD FACSCanto II (BD Biosciences, Franklin Lakes, NJ, USA).

\section{Luciferase Assay}

Luciferase was constructed by ligating oligonucleotides containing the presumed target site of wild-type RNF6 mRNA 3'UTR to the PME1 and XBA1 sites of the pmirGLO vector (Promega, USA). The new vector was named RNF6-WT. The mutant type RNF6 (RNF6 M-MUT) was formed via a QuikChange II Site-Directed Mutagenesis Kit (Agilent, USA). The reporter vector was transfected into miR-26a-5p mimic or treated INS-1 cells using Lipofectamine 2000 for reporter gene detection. A Dual-Luciferase Reporter Assay System (Promega) was used to detect luciferase activity after $24 \mathrm{~h}$ transfection.

\section{Statistical Analysis}

The experiments were repeated three times, and data are expressed as the mean \pm the standard deviation (SD). Intergroup differences were analyzed using the Student's $t$-test and a one-way ANOVA, and Pearson's analysis was used to estimate the correlation between miR-26a-5p and RNF6. All the analyses were conducted using SPSS 22.0 (SPSS, USA) software, and statistical significance was set at $<0.05$.

\section{Results}

\section{T2D Displayed Reduced RNF6 Expression}

GSE25724 from Gene Expression Omnibus (GEO) datasets was used as an mRNA microarray to screen the downregulated genes in human islet samples with T2D. 
With an adj.P $<0.05$, and $\operatorname{logFC}<-2,112$ downregulated genes were screened out. After uploading the downregulated genes to STRING for Gene Ontology (GO) enrichment, RNF6 and GNAS complex locus (GNAS) were predicted to be associated with negative development growth regulation (Figure 1A). qRT-PCR showed that both were expressed at low levels in T2D, and GNAS levels showed no significance in T2D compared with the normal group, so RNF6 was selected as the gene of interest (Figure 1B). Moreover, the in vitro INS1 cell diabetes model was established with a high glucose concentration, which showed a $35 \%$ reduction in RNF6 levels compared to the normal group (Figure 1C). In addition, Western blotting showed that RNF6 levels in the model group were reduced by approximately $25 \%$ compared to the normal group (Figure 1D). Next, highglucose-induced INS- 1 cells were treated with siRNARNF6 and pcDNA3.1-RNF6. qRT-PCR and Western blotting revealed that RNF6 mRNA and protein levels decreased after RNF6 knockdown, while the reverse trend was observed after RNF6 overexpression (Figure 1E and F).

\section{RNF6 Overexpression Promoted Insulin}

\section{Secretion and INS-I Cell Proliferation and Inhibited Apoptosis}

Next, we investigated the functional correlation of RNF6 in T2D and the effects of under- and over-expressed RNF6 on insulin production, proliferation, and apoptosis in INS1 cells. We observed that insulin content in si-RNF6 decreased by approximately $20 \%$ but increased by twofold in the pcDNA3.1-RNF6 group compared to the si-NC or pcDNA3.1 groups (Figure 2A). In addition, under low (3.3 $\mathrm{mM})$ and high $(16.7 \mathrm{mM})$ glucose conditions, low RNF6 expression led to decreased insulin secretion, while overexpression led to increased insulin secretion (Figure 2B). Moreover, RNF6 knockdown reduced INS-1 cell proliferation by approximately $40 \%$, while upregulating RNF6 increased it 1.3-fold (Figure 2C). Furthermore, flow cytometry revealed apoptosis levels, indicating that interference with RNF6 promoted apoptosis, while RNF6 upregulation inhibited apoptosis (Figure 2D).

\section{miR-26a-5p Targeted RNF6 in INS-I Cells} We used three databases (miRDB, TargetScan, and TarBase) to predict the miRNAs targeting RNF6 and identify the RNF6 upstream region. The data showed that
miR-26a-5p and miR-26b-5p were upstream of RNF6 by targeting RNF6 conserved sites (Figure 3A). We measured miR-26a-5p and miR-26b-5p levels in the blood of T2D and healthy subjects and found that miR-26a-5p levels in T2D patients were significantly upregulated compared with the healthy controls. miR-26b-5p levels did not change significantly (Figure 3B). In addition, miR-26a-5p expression was inversely correlated with RNF6 levels (Figure 3C). Moreover, studies at the cellular level revealed that high glucose levels increased miR-26a-5p levels (Figure 3D). This suggests that miR-26a-5p acts as a potential miRNA in T2D. Bioinformatics predictive analysis by TargetScan indicated that RNF6 was a potential target for miR-26a-5p with a putative binding site (Figure 3E). We co-transfected the miR-26a-5p mimic with the RNF6 binding sequence or mutant sequence into INS-1 cells to investigate the regulatory relationship between miR-26a-5p and RNF6. The miR-26a-5p mimic suppressed relative luciferase activity by $55 \%$ in cells co-transfected with RNF6-WT (Figure 3F). Moreover, INS-1 cells were treated with a miR-26a-5p inhibitor or mimic to study the regulatory effect of miR26a-5p on RNF6. Western blot data showed that miR-26a$5 p$ knockdown enhanced RNF6 levels, while miR-26a-5p overexpression suppressed RNF6 levels (Figure 3G). These results suggest that miR-26a-5p targets and downregulates RNF6.

\section{miR-26a-5p Inhibition Reversed the Effect of RNF6 Knockdown on Cell Insulin Secretion, Proliferation, and Apoptosis}

Next, we identified the mechanism by which miR-26a$5 p$ mediates INS-1 cell function by regulating RNF6. ELISA analysis showed that the miR-26a-5p knockdown group's insulin concentration and secretion levels were significantly higher than those of the inhibitor-NC group, and RNF6 silencing reversed the insulin concentration and secretion induced by miR-26a-5p knockdown (Figure 4A and B). Similarly, by inhibiting miR-26a-5p expression, the EDU-positive rate of INS-1 cells increased, while the decreased proliferation ability of RNF6-deficient cells was also recovered (Figure 4C). In addition, flow cytometry indicated that miR-26a-5p interference restrained apoptosis levels, and RNF6 downregulation eliminated the inhibitory action of the miR-26a-5p inhibitor on apoptosis (Figure 4D). These data suggest that silencing miR-26a-5p promotes insulin 

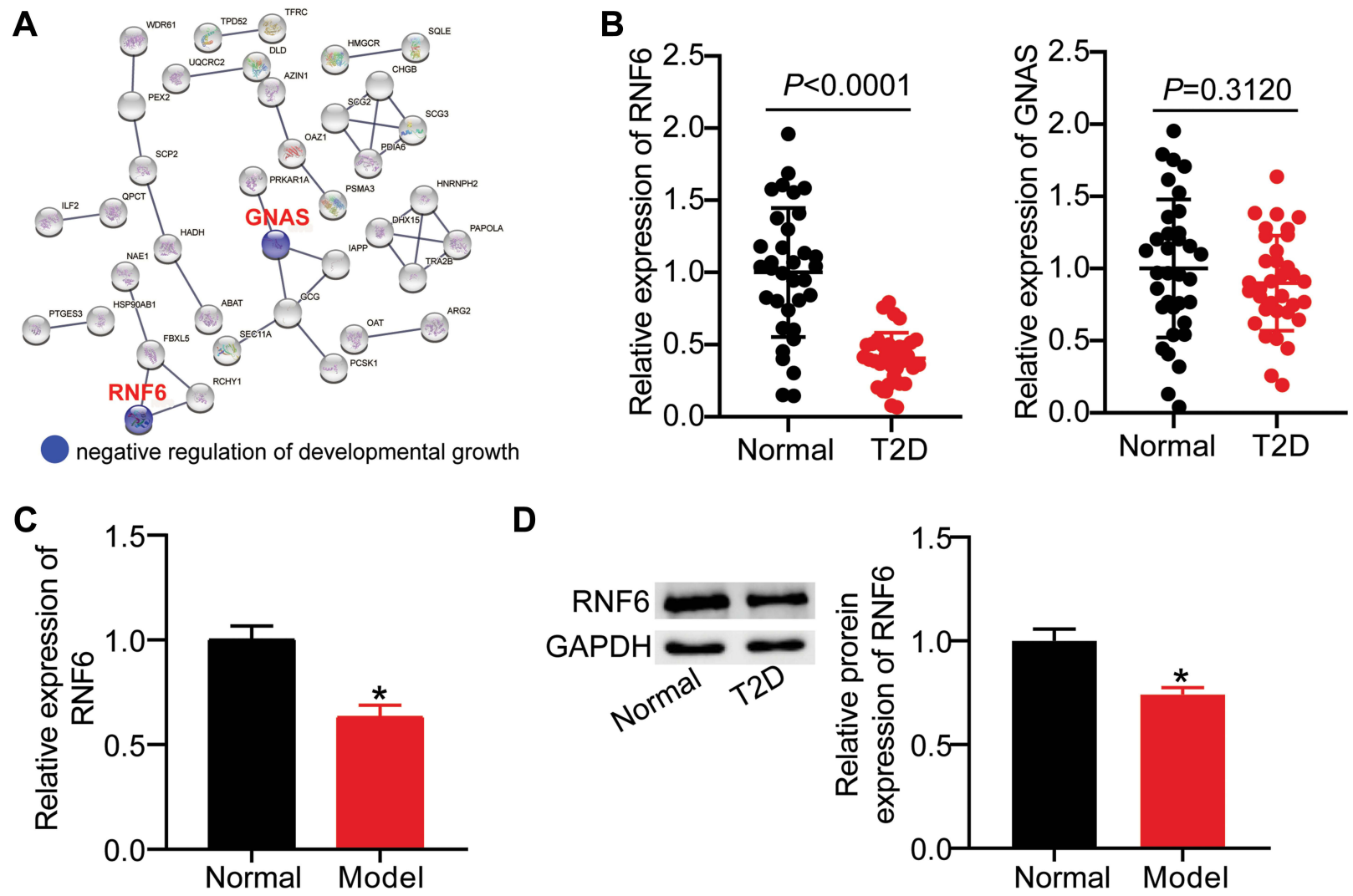

$\mathbf{D}$

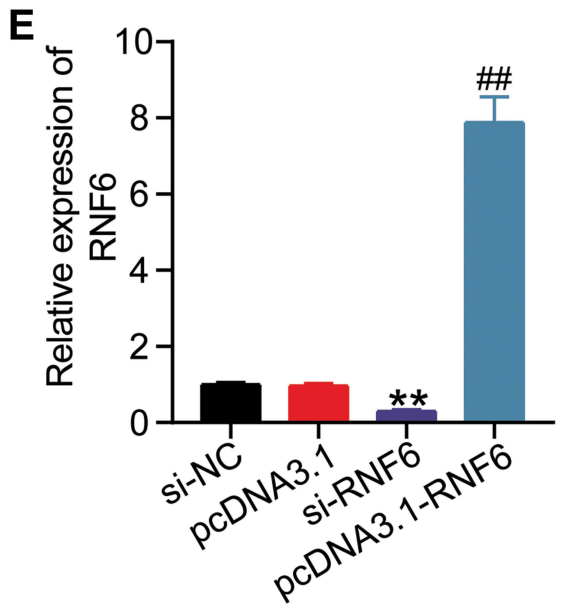

$\mathbf{F}$
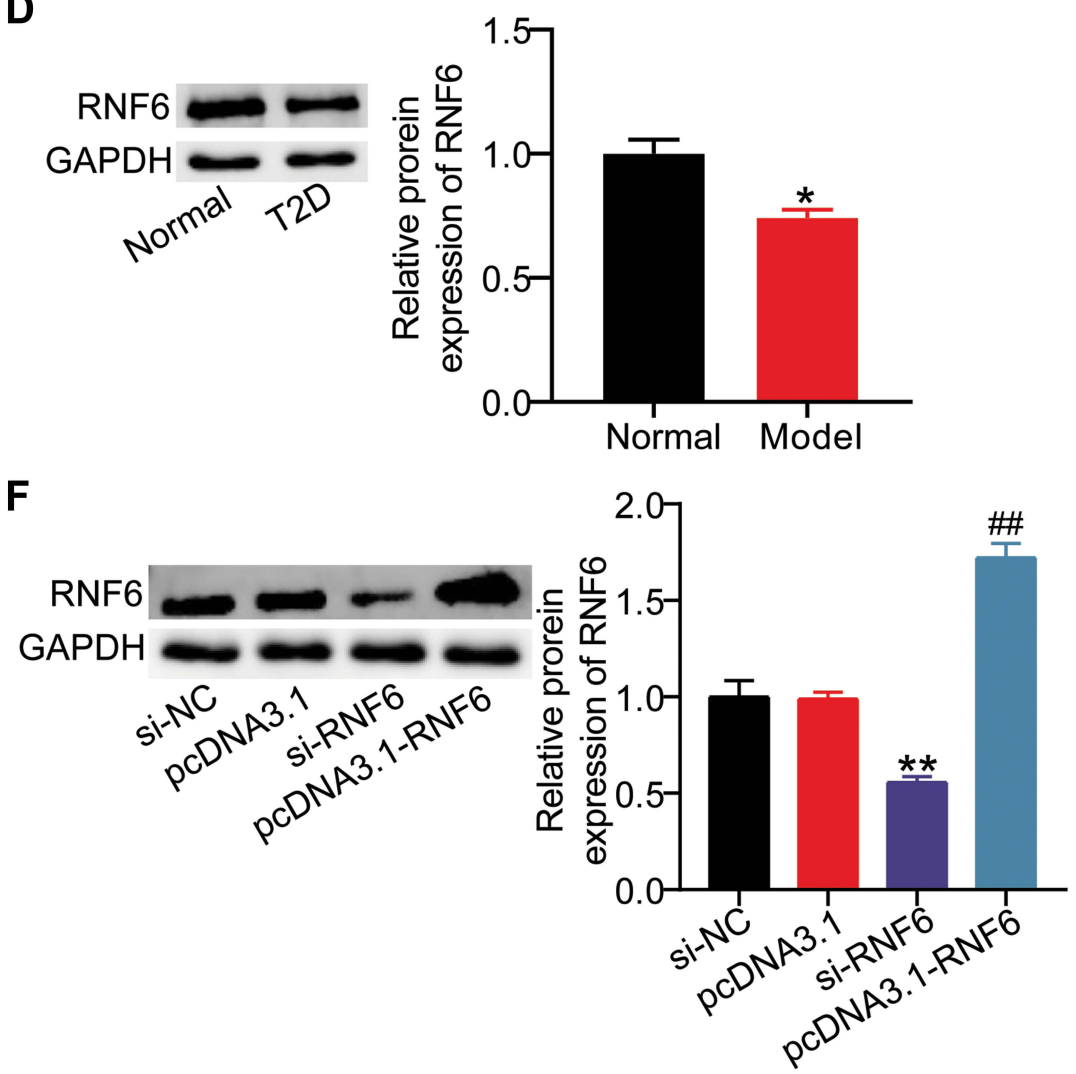

Figure I RNF6 was low expressed in T2D. (A) STRING identified negative regulation of developmental growth including GNAS and RNF6 as the key biological process. (B) The expression levels of RNF6 and GNAS in peripheral blood samples from T2D pregnancies and healthy pregnancies were validated by qRT-PCR. (C) The expression levels of RNF6 in INS-I cells treated with 5.5 (Normal) or 25 mM (Model) glucose were validated by qRT-PCR. *P < 0.05 VS normal. (D) The RNF6 protein level in INS-I cells treated with 5.5 (Normal) or 25 mM (Model) glucose were validated by Western blot. *P $<0.05$ VS normal. (E) The expression levels of RNF6 in INS-I cells treated with RNF6 siRNA or pcDNA3.I were validated by qRT-PCR. **P $<0.00$ I VS si-NC. $\mathrm{P}<0.00 \mathrm{I}$ VS pcDNA3.I. (F) The RNF6 protein level in INS-I cells treated with RNF6 siRNA or pcDNA3.I were validated by Western blot. **P $<0.001$ VS si-NC. ${ }^{\#} \mathrm{P}<0.00$ I VS pcDNA3.I.

secretion and proliferation and inhibits apoptosis by regulating RNF6 in INS-1 cells.

\section{Discussion}

Prolonged $\beta$-cell deficiency affects insulin levels and may have severe glycotoxic effects on pancreatic cells, resulting in impaired insulin secretion. Therefore, it is important to maintain sufficient $\beta$-cell mass to cope with these changes. ${ }^{22}$ Previous research has shown that various miRNAs are involved in $\beta$-cell proliferation, apoptosis, and insulin secretion and may play a role in pancreatic development and diabetes. ${ }^{23}$ For example, PED/PEA-15 overexpression in T2D controls $\beta$-cell mass, alters caspase- 3 activation, and apoptotic gene expression. ${ }^{24}$ FGF21 protects against 

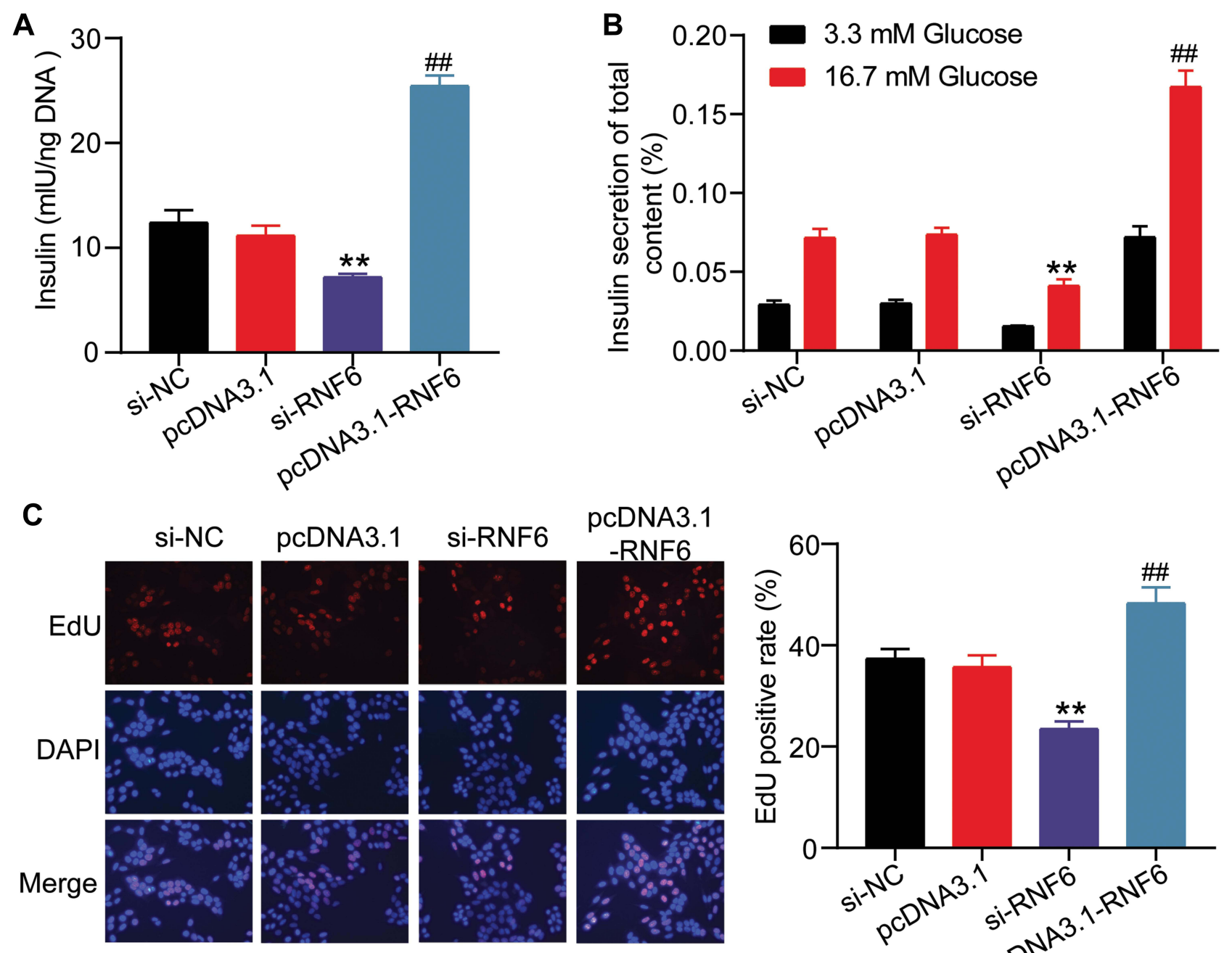

pcDNA3. 1
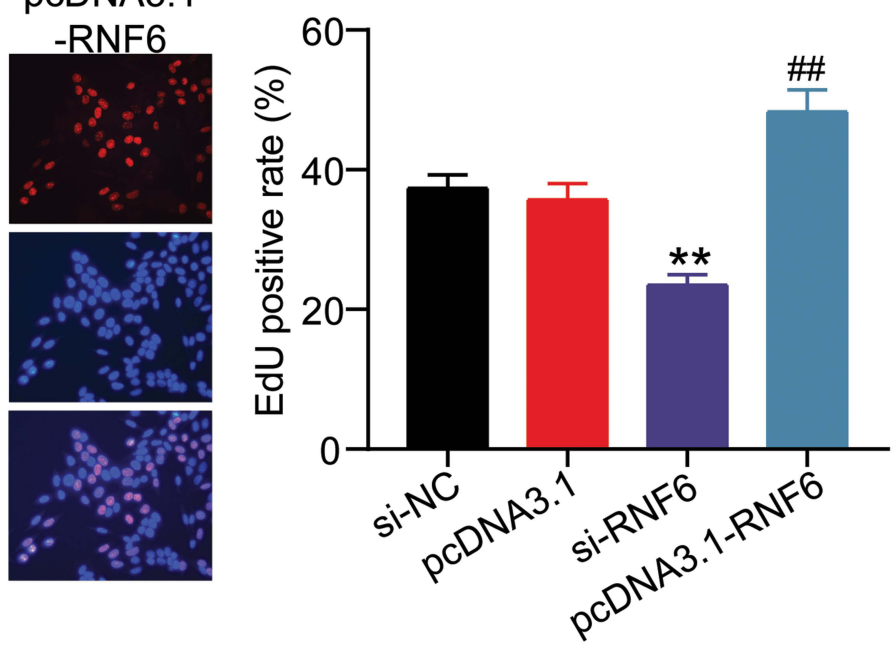

D
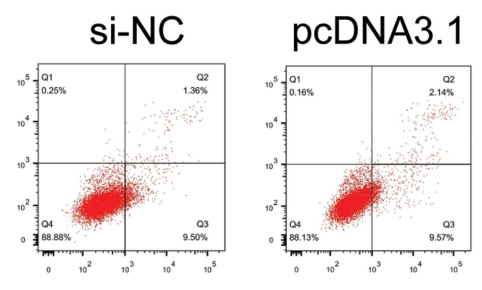

si-RNF6
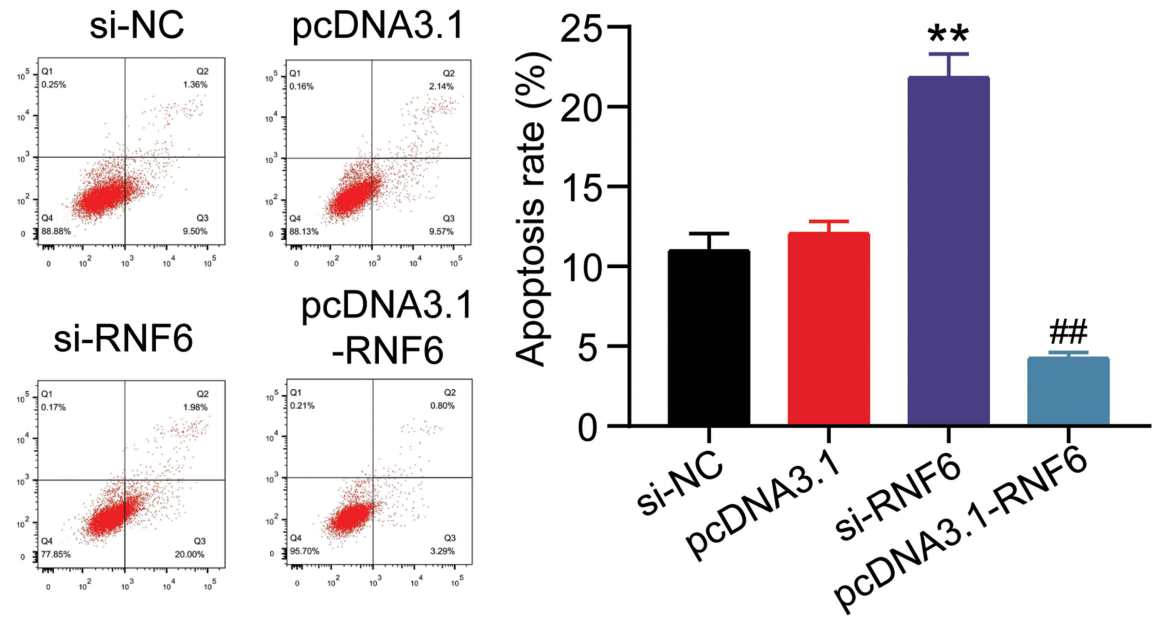

Figure 2 Overexpression of RNF6 promoted insulin secretion and proliferation of INS-I cells, inhibited apoptosis. (A) Insulin content was determined by ELISA assay in INS-I cells treated with RNF6 siRNA or pcDNA3.I. (B) insulin secretion was determined by ELISA assay in INS-I cells treated with RNF6 siRNA or pcDNA3.I. (C) cell proliferation was determined by EdU assay in INS-I cells treated with RNF6 siRNA or pcDNA3.I. (D) cell apoptosis was determined by flow cytometry assay in INS-I cells treated with RNF6 siRNA or pcDNA3.I. **P $<0.001$ VS si-NC. ${ }^{\#} \mathrm{P}<0.001$ VS pcDNA3.I.

lipotoxicity-induced $\beta$-cell dysfunction and apoptosis by decreasing lipid accumulation and reducing cell death in islet cells. ${ }^{25}$
Several genes that increase susceptibility to diabetes are still incomplete and unidentified. ${ }^{26}$ The accumulated literature indicates that the $\beta$-cell gene, RNF6, is 
A

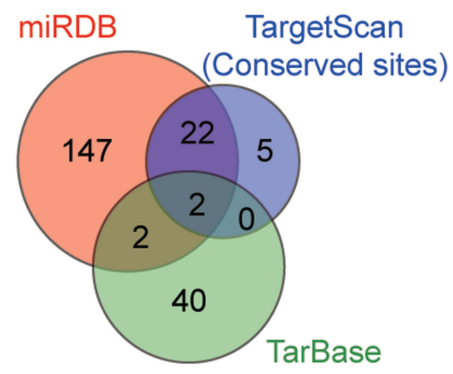

C

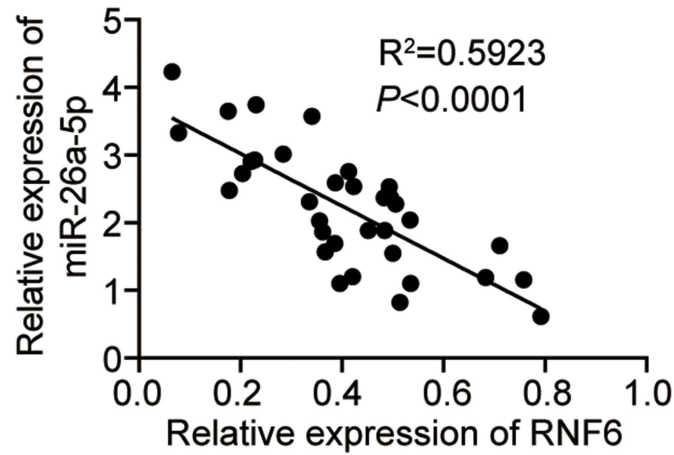

B

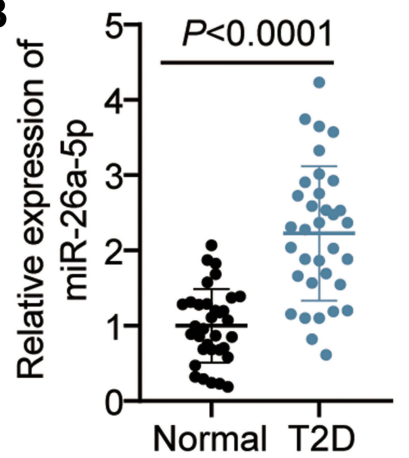

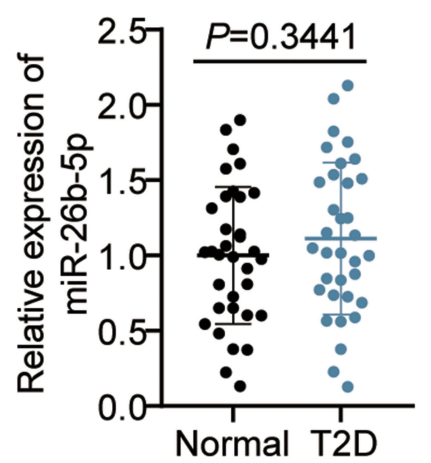

E

Position 443-450 of RNF6 3' UTR: $\quad 5^{\prime}$...UAGAUAUACUUAAGUUACUUGAA...

rno-miR-26a-5p: $\quad$ 3' UCGGAUAGGACCUAAUGAACUU

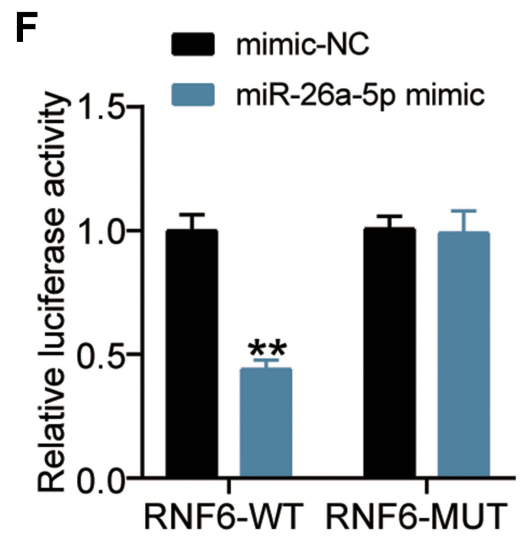

G

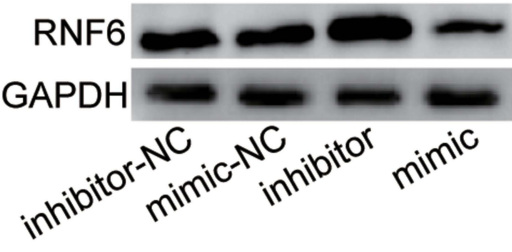

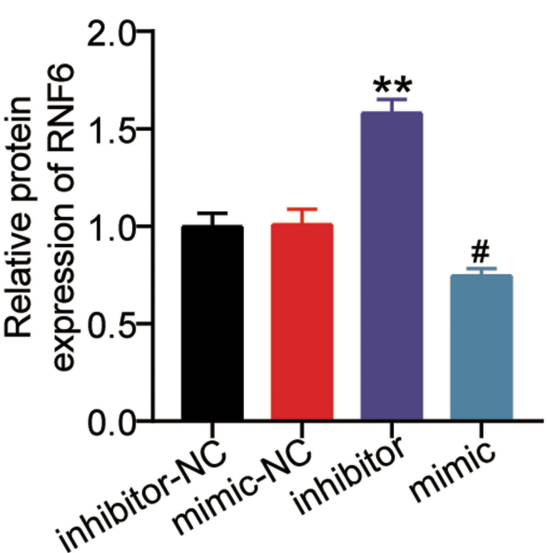

Figure 3 miR-26a-5p targeted RNF6 in INS-I cells. (A) miR-26a-5p and miR-26b-5p were predicted as the upstream of RNF6 in miRDB, TargetScan and TarBase. (B) The expression levels of miR-26a-5p and miR-26b-5p in peripheral blood samples from T2D pregnancies and healthy pregnancies were validated by $q R T-P C R$. (C) The correlation between miR-26a-5p and RNF6 in peripheral blood samples from T2D pregnancies. (D)The expression level of miR-26a-5p in INS-I cells treated with 5.5 (Normal) or 25 $\mathrm{mM}$ (Model) glucose were validated by qRT-PCR. **P $<0.001$ VS normal. (E) Sequence comparison between miR-26a-5p and RNF6 indicating their putative binding region. (F) Dual-luciferase reporter assay of INS-I cells co-transfected with RNF6-WT/MUT and mimic-NC/miR-26a-5p mimic. **P $<0.00$ I VS mimic-NC. (G) The RNF6 protein level in INS-I cells treated with miR-26a-5p mimic or inhibitor were validated by Western blot. **P $<0.00$ I VS inhibitor-NC. ${ }^{*} \mathrm{P}<0.05$ VS mimic-NC.

specifically expressed in one or more endocrine cell types. ${ }^{27}$ Here, we investigated the role of RNF6 in T2D and glucose-treated $\beta$-cells. RNF6 was downregulated in T2D serum. Furthermore, we found that RNF6 upregulation increased proliferation and insulin content, induced insulin secretion, and inhibited apoptosis, while RNF6 downregulation inhibited $\beta$-cell function. These results demonstrate for the first time the RNF6 therapeutic effect on $\mathrm{T} 2 \mathrm{D}$ by protecting pancreatic $\beta$-cells. This suggests that RNF6 may be a predictive gene for diabetes.

Although many miRNAs have been found to act in T2D, such as miR-3666 to reduce insulin sensitivity ${ }^{28}$ 


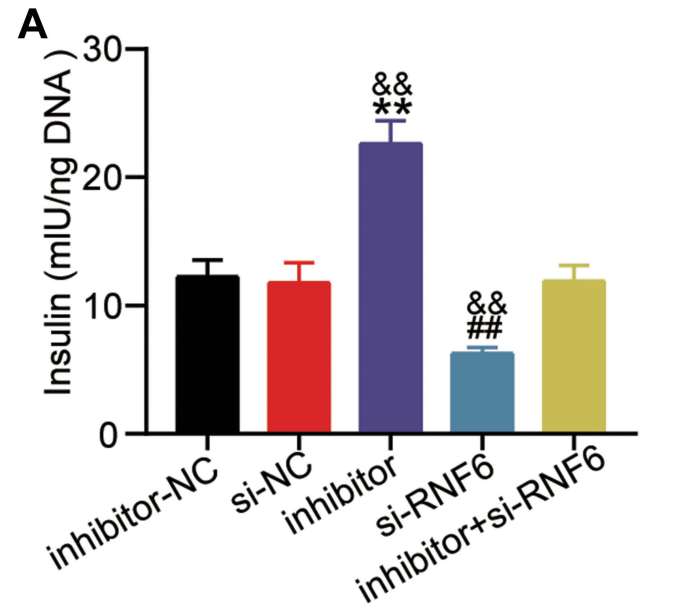

C

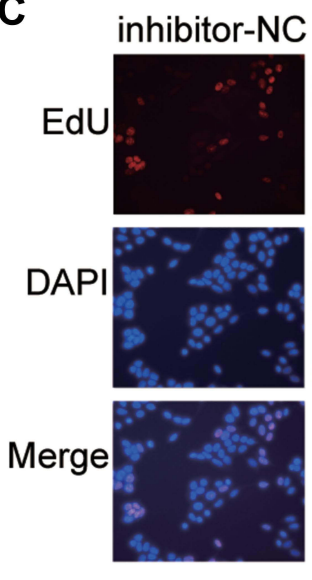

D inhibitor-NC
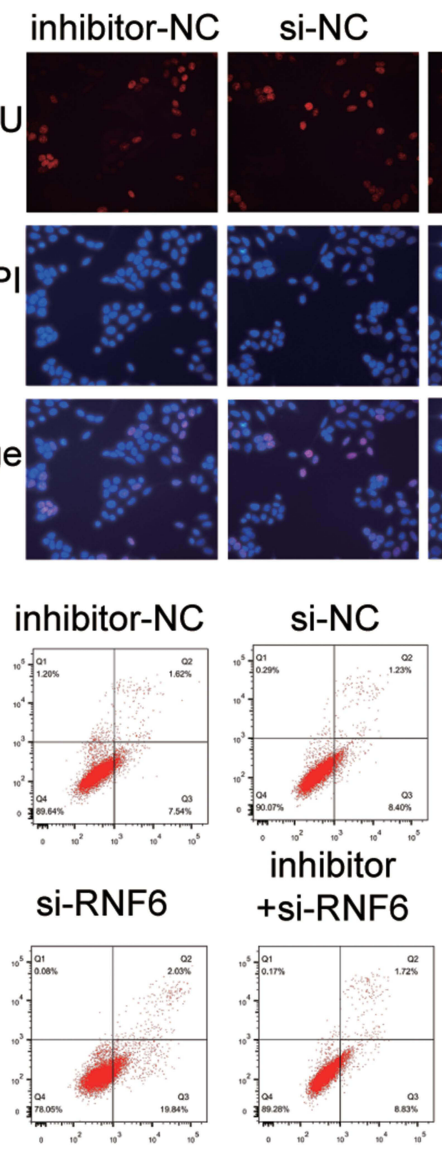

inhibitor +si-RNF6

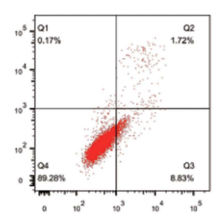

B

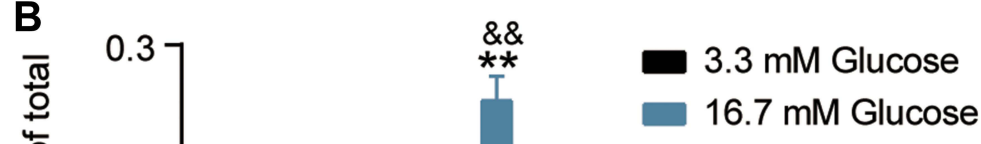

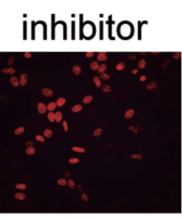
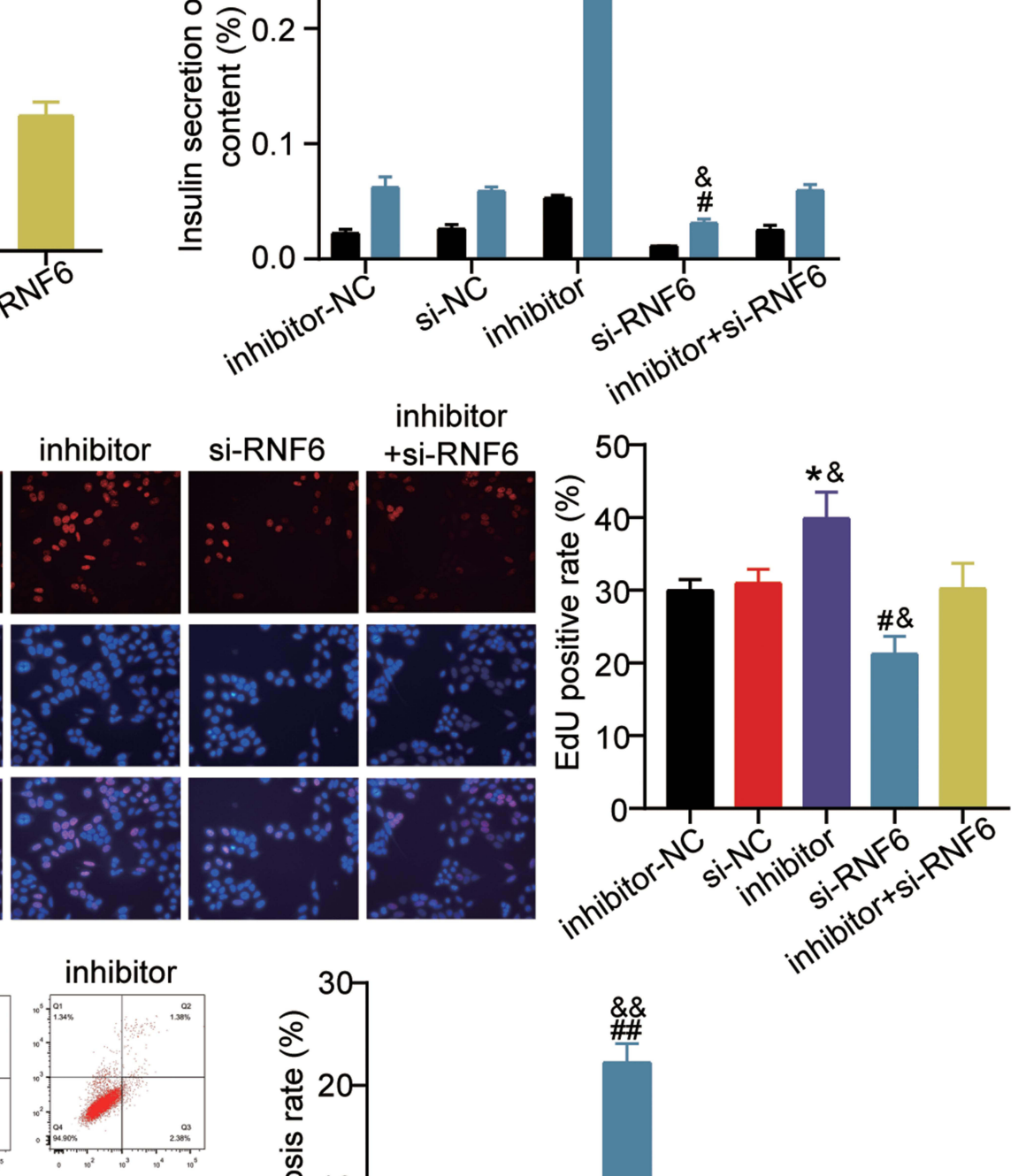

inhibitor

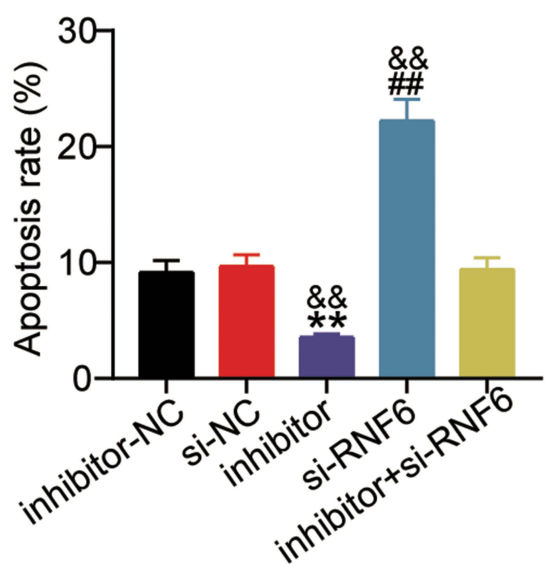

Figure 4 miR-26a-5p inhibition reversed the effect of RNF6 knockdown on cell insulin secretion, proliferation and apoptosis. (A) Insulin content was determined by ELISA assay in INS-I cells treated with miR-26a-5p inhibitor or RNF6 siRNA. (B) insulin secretion was determined by ELISA assay in INS-I cells treated with miR-26a-5p inhibitor or RNF6 siRNA. (C) cell proliferation was determined by EdU assay in INS-I cells treated with miR-26a-5p inhibitor or RNF6 siRNA. (D) cell apoptosis was determined by flow cytometry assay in INS-I cells treated with miR-26a-5P inhibitor or RNF6 siRNA. ${ }^{* P}<0.05,{ }^{* * P}<0.00 I$ VS inhibitor-NC. ${ }^{\#} P<0.05$, ${ }^{\# \#} P<0.00 I$ VS si-NC. ${ }^{\&} P<0.05$, \&\& $P<0.00$ I VS inhibitor+si-RNF6.

and miR-375 to normalize $\beta$-cell quality and function, ${ }^{29}$ the exact role and potential mechanism of miRNA in T2D $\beta$-cells still need to be further studied. In this study, miR26b-5p was shown to be upregulated in T2D and high glucose-treated $\beta$-cells. Furthermore, RNF6 was negatively correlated with miR-26b-5p in T2D and was adversely regulated by miR-26b-5p in $\beta$-cells. In addition, RNF6 was revealed to be a miR-26b-5p target through a bioinformatics website and luciferase assay. Kei et al found similar results of miR-26b targeting RNF6. ${ }^{17}$ 
Functional studies have shown that interference with miR$26 b-5 p$ increased the survival and insulin content of $\beta$-cells treated with high glucose, induced insulin secretion, and impeded apoptosis. The rescue experiment revealed that miR-26b-5p knockdown achieved its therapeutic effect on T2D by acting on RNF6.

Although the effects of miR-26b-5p/RNF6 on $\beta$-cell insulin content, secretion, survival, and apoptosis have been studied, our study has some limitations. For example, we did not establish a T2D animal model to explore the effects of miR-26b-5p/RNF6 on insulin secretion in vivo. In addition, the upstream and downstream mechanisms of miR-26b-5p/RNF6 in T2D need further investigation.

\section{Conclusion}

This study showed that RNF6 is downregulated in T2D. RNF6 overexpression enhanced INS-1 cell proliferation and insulin production and inhibited apoptosis. miR-26b$5 p$ aggravates T2D by targeting RNF6 to regulate INS-1 cell function. The results of our study provide some value for the role of miR-26b-5p/RNF6 in T2D.

\section{Data Sharing Statement}

The datasets used and/or analyzed during the current study are available from the corresponding author on reasonable request.

\section{Ethics Approval and Informed Consent}

The present study was approved by the Ethics Committee of the Wuhan University Zhongnan Hospital (Wuhan, China). The processing of clinical tissue samples is in strict compliance with the ethical standards of the Declaration of Helsinki. All patients signed written informed consent.

\section{Consent for Publication}

Consent for publication was obtained from the participants.

\section{Consent to Participate}

All patients signed written informed consent.

\section{Funding}

The authors disclosed receipt of the following financial support for the research, authorship, and/or publication of this article: This work was supported by National Natural Science Foundation of China (81970718).

\section{Disclosure}

The authors declare that they have no conflict of interests.

\section{References}

1. Godfrey JD, Morton JP, Wilczynska A, Sansom OJ, Bushell MD. MiR-142-3p is downregulated in aggressive p53 mutant mouse models of pancreatic ductal adenocarcinoma by hypermethylation of its locus. Cell Death Dis. 2018;9(6):644. doi:10.1038/s41419-018-0628-4

2. Prasun P. Role of mitochondria in pathogenesis of type 2 diabetes mellitus. J Diabetes Metab Disord. 2020;19(2):2017-2022. doi:10.1007/s40200-020-00679-x

3. Saeedi P, Petersohn I, Salpea P, et al. Global and regional diabetes prevalence estimates for 2019 and projections for 2030 and 2045: results from the International Diabetes Federation Diabetes Atlas, 9(th) edition. Diabetes Res Clin Pract. 2019;157:107843. doi:10.1016/j.diabres.2019.107843

4. Liu J, Sun X, Zhang FL, et al. Clinical potential of extracellular vesicles in type 2 diabetes. Front Endocrinol (Lausanne). 2020;11:596811. doi:10.3389/fendo.2020.596811

5. Prasad RB, Groop L. Genetics of type 2 diabetes-pitfalls and possibilities. Genes. 2015;6(1):87-123. doi:10.3390/genes6010087

6. Liu L, Zhang Y, Wong CC, et al. RNF6 promotes colorectal cancer by activating the $\mathrm{Wnt} / \beta$-catenin pathway via ubiquitination of TLE3. Cancer Res. 2018;78(8):1958-1971. doi:10.1158/0008-5472.Can-17-2683

7. Lipkowitz S, Weissman AM. RINGs of good and evil: RING finger ubiquitin ligases at the crossroads of tumour suppression and oncogenesis. Nat Rev Cancer. 2011;11(9):629-643. doi:10.1038/nrc3120

8. Cai J, Xiong Q, Jiang X, Zhou S, Liu T. RNF6 facilitates metastasis and radioresistance in hepatocellular carcinoma through ubiquitination of FoxA1. Exp Cell Res. 2019;374(1):152-161. doi:10.1016/j. yexcr.2018.11.019

9. Qiu Y, Zhu H, Xu D, et al. RING-finger protein 6 enhances c-Mycmediated Warburg effect by promoting MAD1 degradation to facilitate pancreatic cancer metastasis. Am J Cancer Res. 2021;11 (5):2025-2043.

10. Jin G, Wang Q, Pei X, et al. mRNAs expression profiles of high glucose-induced memory in human umbilical vein endothelial cells. Diabetes Metabol Syndr Obes. 2019;12:1249-1261. doi:10.2147/ dmso.S206270

11. Lyssenko V, Laakso M. Genetic screening for the risk of type 2 diabetes: worthless or valuable? Diabetes Care. 2013;36(Suppl2): S120-6. doi:10.2337/dcS13-2009

12. Fabian MR, Sonenberg N, Filipowicz W. Regulation of mRNA translation and stability by microRNAs. Annu Rev Biochem. 2010;79:351-379. doi:10.1146/annurev-biochem-060308-103103

13. Saliminejad K, Khorram Khorshid HR, Soleymani Fard S, Ghaffari SH. An overview of microRNAs: biology, functions, therapeutics, and analysis methods. $J$ Cell Physiol. 2019;234 (5):5451-5465. doi:10.1002/jcp.27486

14. Stępień E, Durak-Kozica M, Kamińska A, et al. Circulating ectosomes: determination of angiogenic microRNAs in type 2 diabetes. Theranostics. 2018;8(14):3874-3890. doi:10.7150/thno.23334

15. Demirsoy İH, Ertural DY, Balci Ş, et al. Profiles of circulating miRNAs following metformin treatment in patients with type 2 diabetes. J Med Biochem. 2018;37(4):499-506. doi:10.2478/jomb2018-0009

16. Li HX, Li XH, Jiang J, Shi PX, Zhang XG, Tian M. Effect of miR-26b on gestational diabetes mellitus in rats via PI3K/Akt signaling pathway. Eur Rev Med Pharmacol Sci. 2020;24(4):1609-1615. doi:10.26355/eurrev_202002_20335

17. Takahashi K, Tatsumi N, Fukami T, Yokoi T, Nakajima M. Integrated analysis of rifampicin-induced microRNA and gene expression changes in human hepatocytes. Drug Metab Pharmacokinet. 2014;29(4):333-340. doi:10.2133/dmpk.dmpk-13-rg-114 
18. Grimaldi A, Heurtier A. [Diagnostic criteria for type 2 diabetes]. Rev Prat. 1999;49(1):16-21. French.

19. Livak KJ, Schmittgen TD. Analysis of relative gene expression data using real-time quantitative PCR and the 2(-Delta Delta C(T)) method. Methods. 2001;25(4):402-408. doi:10.1006/meth.2001.1262

20. Naujok O, Bandou Y, Shikama Y, Funaki M, Lenzen S. Effect of substrate rigidity in tissue culture on the function of insulin-secreting INS-1E cells. J Tissue Eng Regen Med. 2017;11(1):58-65. doi:10.1002/term. 1857

21. Kao SY, Shyu JF, Wang HS, et al. Comparisons of differentiation potential in human mesenchymal stem cells from Wharton's jelly, bone marrow, and pancreatic tissues. Stem Cells Int. 2015;2015:306158. doi:10.1155/2015/306158

22. Armstrong D. Preface. Advanced protocols in oxidative stress III. Methods Mol Biol. 2015;1208:v-vi. doi:10.1007/978-1-4939-1441-8

23. Chakraborty C, George Priya Doss C, Bandyopadhyay S. miRNAs in insulin resistance and diabetes-associated pancreatic cancer: the 'minute and miracle' molecule moving as a monitor in the 'genomic galaxy'. Curr Drug Targets. 2013;14(10):1110-1117. doi:10.2174/ 13894501113149990182
24. Fiory F, Spinelli R, Raciti GA, et al. Targetting PED/PEA-15 for diabetes treatment. Expert Opin Ther Targets. 2017;21(6):571-581. doi:10.1080/14728222.2017.1317749

25. Xie T, So WY, Li XY, Leung PS. Fibroblast growth factor 21 protects against lipotoxicity-induced pancreatic $\beta$-cell dysfunction via regulation of AMPK signaling and lipid metabolism. Clin Sci. 2019;133 (19):2029-2044. doi:10.1042/cs20190093

26. Himanshu D, Ali W, Wamique M. Type 2 diabetes mellitus: pathogenesis and genetic diagnosis. J Diabetes Metab Disord. 2020;19 (2):1959-1966. doi:10.1007/s40200-020-00641-x

27. Fang Z, Weng C, Li H, et al. Single-cell heterogeneity analysis and CRISPR screen identify key $\beta$-cell-specific disease genes. Cell Rep. 2019;26(11):3132-3144.e7. doi:10.1016/j.celrep.2019.02.043

28. Tan J, Tong A, Xu Y. Pancreatic $\beta$-cell function is inhibited by miR3666 in type 2 diabetes mellitus by targeting adiponectin. Braz J Med Biol Res. 2019;52(6):e8344. doi:10.1590/1414-431x20198344

29. Latreille M, Herrmanns K, Renwick N, et al. miR-375 gene dosage in pancreatic $\beta$-cells: implications for regulation of $\beta$-cell mass and biomarker development. $J$ Mol Med. 2015;93(10):1159-1169. doi:10.1007/s00109-015-1296-9

\section{Publish your work in this journal}

Diabetes, Metabolic Syndrome and Obesity: Targets and Therapy is an international, peer-reviewed open-access journal committed to the rapid publication of the latest laboratory and clinical findings in the fields of diabetes, metabolic syndrome and obesity research. Original research, review, case reports, hypothesis formation, expert opinion and commentaries are all considered for publication. The manuscript management system is completely online and includes a very quick and fair peer-review system, which is all easy to use. Visit http://www.dovepress.com/testimonials.php to read real quotes from published authors. 